\title{
Effectiveness of robot-assisted gait training on freezing of gait in people with Parkinson disease: evidence from a literature review
}

\author{
Mohammad Alwardat ${ }^{1,2, *}$, Mohammad Etoom ${ }^{3}$ \\ 'Department of Systems Medicine, University of Rome "Tor Vergata", Rome, Italy \\ ${ }^{2}$ Neuroscience PhD School, University of Rome "Tor Vergata", Rome, Italy \\ ${ }^{3}$ Department of Physical Therapy, Al-Isra University, Amman, Jordan
}

The aim of this review was to evaluate, summarize and discuss the available literature concerning the effect of robot-assisted gait training (RAGT) on patient with Parkinson disease (PD) and freezing of gait (FOG). A comprehensive literature search was conducted utilizing of MEDLINE, Embase, Scopus, Web of Science, PEDro (Physiotherapy Evidence Database), and the Cochrane Review. Search terms used included 'Parkinson disease,' 'Freezing of gait,' 'RAGT,' 'robot-assisted gait training,' 'Locomotor rehabilitation,' 'gait trainer,' and 'robotics assisted gait training.' A total of 4 studies were evaluated, but these studies were primarily of low-level evidence. All the 4 studies noted positive outcomes with using RAGT on FOG. No adverse events or side effects that occurred during and/or after the interventions. While the current literature generally offers support for the use of RAGT for FOG treatment, there is a paucity of strong evidence to support its widespread use. The increasing availability of RAGT technology offers the potential for engaging therapy in FOG rehabilitation, but its utility remains uncertain given the limited studies available at this time.

Keywords: Parkinson disease, Robot-assisted gait training, Freezing of gait, Physiotherapy, Rehabilitation

\section{INTRODUCTION}

Freezing of gait (FOG) is one of the most disturbing and least poorly understood gait phenomenon in people with Parkinson disease (PD) (Nutt et al., 2011). In the early PD stage, about 20\% of patients report FOG, but the prevalence of this phenomenon raises up to $50 \%$ in the advanced disease stages (Giladi et al., 2001). People with PD (PwPD) are experience FOG in various situations: when starting to walk, during turning, when approaching a narrow space, and just before reaching destination (Frazzitta et al., 2009). FOG is highly impairs mobility, causes falls, limits activities of daily living and reduces quality of life (Bloem et al., 2004; De Boer et al., 1996; Kerr et al., 2010). Since the pathogenesis of FOG is still unclear, no available treatment for this disabling phenomenon (Nutt et al., 2011). The pharmacolog- ical treatment is disappointing: PwPD and FOG in "off" states gained more benefit from an increase in levodopa dosage, this was not observed in the same patient in "on" states (Schaafsma et al., 2003). In addition, the surgical treatment (deep brain stimulation) and levodopa, are insufficient for treating FOG (Ferraye et al., 2010).

The nonpharmacological interventions like rehabilitation are a possible treatment for gait disorders in PwPD. Several physical therapy (PT) approaches have been employed in PD rehabilitation protocols for helping patients overcome PD symptoms and FOG episodes (Alwardat, 2018a; Chang et al., 2017; Frazzitta et al., 2009; Killane et al., 2015; Pelosin et al., 2018). These approaches include transcranial direct current stimulation, auditory and visual cueing training and virtual reality training (Chang et al., 2017; Frazzitta et al., 2009; Killane et al., 2015). Many studies have
${ }^{*}$ Corresponding author: Mohammad Alwardat (iD https://orcid.org/0000-0003-2106-4524

Neuroscience PhD School, Faculty of Medicine and Surgery, University of Rome

"Tor Vergata”, Via Montpellier, 1, 00133, Rome, Italy

E-mail: wardatm1@gmail.com

Received: December 14, 2018 / Accepted: January 15, 2019
This is an Open Access article distributed under the terms of the Creative Commons Attribution Non-Commercial License (http://creativecommons.org/licenses/by-nc/4.0/) which permits unrestricted non-commercial use, distribution, and reproduction in any medium, provided the original work is properly cited. 
shown the efficacy of PT interventions at improving specific impairments and functional limitations in PwPD (Alwardat et al., 2018b; Goodwin et al., 2008; Mehrholz et al., 2010). In particular, they have shown that PT interventions can reduce the incidence and duration of FOG episodes (Chang et al., 2017; Frazzitta et al., 2009; Killane et al., 2015; Pelosin et al., 2018). However, to date, findings are not univocal, and long-term consolidation of performance improvement needs to be investigated in future studies.

There is growing interest in using robot-assisted gait training (RAGT) as a new PT technology for meeting the therapeutic gaps associated with PD and FOG. In our recent systematic review and meta-analysis, RAGT showed better outcomes than conventional interventions on some motor aspects in PD (Alwardat et al., 2018b). In line with our results, previous studies in PD and other neurological disease such as stroke and spinal cord injury that used RAGT showed significant improvements in gait and balance, compared with conventional rehabilitation programs (Furnari et al., 2017; Picelli et al., 2012; Sale et al., 2012). However, to our knowledge, no systematic review and/or review of the literature investigates the effect of RAGT on PwPD and FOG. Therefore, the objectives of this review are to evaluate, summarise and discuss the available literature concerning the effect of RAGT on FOG.

\section{MATERIALS AND METHODS}

A comprehensive literature search was conducted utilizing of MEDLINE, Embase, Scopus, Web of Science, PEDro (Physiotherapy Evidence Database), and the Cochrane Review. The search included studies published from January 1990 to October 2018. Search terms used included 'Parkinson disease, 'Freezing of gait,' 'RAGT,' 'robot-assisted gait training,' 'Locomotor rehabilitation training,' 'gait trainer,' and 'robotics assisted gait training.' Search strategies were developed for each database using both free-text terms and the MeSH (medical subject headings). The reference lists of relevant articles were screened for potential related articles.

Inclusion was dependent on the following criteria: participants: PwPD diagnosed according to the Criteria of UK Parkinson's Disease Brain Bank or confirmed by medical diagnosis, and presence with FOG. Any study investigated the effects of RAGT on FOG. The primary outcomes are Freezing of Gait-Questionnaire (FOG-Q) and Unified Parkinson Disease Rating Scale Part III (UPDRS III). Secondary outcomes include balance, gait, and posture measurements. Studies including participants with neurolog- ical conditions other than PD were excluded. Studies in language other than English, systematic reviews and meta-analyses were not included in the current review. Data related to study design, duration and frequency of the RAGT intervention program, sample size, and outcome measures were extracted for qualitative analysis in accordance with the Preferred Reporting Items for Systematic Reviews and Meta- Analyses guidelines (PRISMA).

\section{RESULTS}

Of the 1948 papers reviewed, four studies (Barbe et al., 2013; Lo et al., 2010; Pilleri et al., 2015; Ustinova et al., 2011) were relevant to the specified criteria, detailing the use of RAGT to treat FOG in PwPD. Retrieved data are presented in Table 1, their year of publication spanning from 2010 to 2015. Studies varied in size and outcome measures, and most had poor methodological quality. The total number of participants was 26, and age range was 63-67 years and the range of PD duration was 6.1811.5 years. $\mathrm{PD}$ severity according to $\mathrm{H} \& \mathrm{Y}$ staging scale ranged from 2.5 to 3.5 in all studies, except one study (Lo et al., 2010), the authors did not report the H\&Y stage. Mini Mental Status Examination used in 2 of the included studies (Barbe et al., 2013; Pilleri et al., 2015) to eliminate potential cognitive impairment. All the included studies reported that the participant trained and were tested during their usual medication ('ON' medication phase). Two studies assessed the long term effect of RAGT, Barbe et al., 2013 assessed after 6 weeks, and Ustinova et al. (2011) assessed the effect after 15 weeks. All studies used RAGT as an experimental intervention supervised by a physiotherapist. The RAGT devices used in the included studies were Lokomat (Barbe et al., 2013; Lo et al., 2010; Ustinova et al., 2011), except Pilleri et al. (2015) did not report the RAGT device. RAGT was performed at speed ranged from 0.7 to $2.5 \mathrm{~km} / \mathrm{hr}$ in all the included studies. The frequency of the treatment ranged from one to three times per week during 'on' medication phase in all included studies. The treatment duration (in terms of session duration) ranged from 30 to $40 \mathrm{~min}$. The summary of the interventions is presented in Table 1. Most of the studies were of low-level evidence including three case studies (Barbe et al., 2013; Lo et al., 2010; Ustinova et al., 2011) and one uncontrolled experimental study (Pilleri et al., 2015).

\section{Effect of RAGT on FOG}

All the included studies in this review concluded positive outcomes with using RAGT to treat FOG. Lo et al. (2010) examined 
Table 1. The summary and characteristics of the included studies

\begin{tabular}{|c|c|c|c|c|c|c|c|}
\hline Study & Study type & $\begin{array}{l}\text { Participants, demographical, } \\
\text { and clinical data }\end{array}$ & $\begin{array}{c}\text { Outcome } \\
\text { measurements }\end{array}$ & Device type & $\begin{array}{l}\text { Duration of } \\
\text { intervention }\end{array}$ & $\begin{array}{l}\text { Total No. of } \\
\text { sessions }\end{array}$ & Main results \\
\hline Lo et al. (2010) & Case study & $\begin{array}{l}\mathrm{n}=4 ; \text { Age: } 63.25 \pm 17.29 \mathrm{yr} ; \\
\text { PD duration: } 6.18 \pm 5.91 \mathrm{yr} ; \\
\text { UPDRS-III: } 20.75 \pm 9.07\end{array}$ & $\begin{array}{l}\text { FOG-0, } \\
\text { FOG falls diary, } \\
\text { vFOG }\end{array}$ & Lokomat & $\begin{array}{l}30 \min \times \\
2 / w k \times 5 \text { wk }\end{array}$ & 10 & $\begin{array}{l}\text { Significant improvement in frequency of } \\
\text { freezes per day } \\
\text { FOG-O scores and FOG severity } \\
\text { significantly improved after RAGT. }\end{array}$ \\
\hline $\begin{array}{l}\text { Barbe et al. } \\
\text { (2013) }\end{array}$ & Case study & $\begin{array}{l}\mathrm{n}=3 \text {; Age: } 64 \pm 4.36 \mathrm{yr} ; \\
\text { Pd duration: } 7.67 \pm 2.08 \text {; } \\
\text { UPDRS-III: } 31 \pm 13.89\end{array}$ & FOG-0 & Lokomat & $30 \mathrm{~min}$ & 10-12 & $\begin{array}{l}\text { FOG significantly improved after RAGT } \\
\text { intervention. } \\
\text { No significant improvement observed } \\
\text { after } 6 \text { weeks of RAGT interventions. }\end{array}$ \\
\hline $\begin{array}{l}\text { Ustinova et al. } \\
\text { (2011) }\end{array}$ & Case study & $\begin{array}{l}\text { n= 1; Age: } 67 \text { yr; PD } \\
\text { duration: 8; UPDRS-III: } 40\end{array}$ & UPDRS item 14 & Lokomat & $\begin{array}{l}25-40 \min \times \\
3 / w k \times 2 w k\end{array}$ & 6 & $\begin{array}{l}\text { Walking activities significantly improved } \\
\text { and the occurrence of freezing episodes } \\
\text { is reduced after RAGT interventions. }\end{array}$ \\
\hline $\begin{array}{l}\text { Pilleri et al. } \\
\text { (2015) }\end{array}$ & $\begin{array}{l}\text { Pilot, } \\
\text { uncontrolled } \\
\text { study }\end{array}$ & $\begin{array}{l}\text { n= 18; Age: } 64.5 \text { yr; PD } \\
\text { duration: } 11.5 ; \text { UPDRS-III: } \\
24.235\end{array}$ & FOG-0 & NR & $\begin{array}{l}30 \min \times \\
5 / w k \times 3 w k\end{array}$ & 15 & $\begin{array}{l}\text { FOG, gait parameters and functional } \\
\text { balance improved significantly after } \\
\text { RAGT interventions. }\end{array}$ \\
\hline
\end{tabular}

FOG, freezing of gait; FOG-Q, freezing of gait questionnaire; vFOG, visual freezing of gait; NR, not reported; PD, Parkinson disease; RAGT, robot-assisted gait training; UPDRS-III, Unified Parkinson's Disease Rating scale-motor part III.

the potential effect of continuous physical cueing using robot-assisted sensorimotor gait training on reducing FOG episodes and improving gait. Four subjects participated in this pilot study, the median age was 63.25 years, disease duration was 6.18 years and median UPDRS III score was 20.5. Participants trained twice a week for five weeks in an "on" phase medication, and each training session on the RAGT (Lokomat, Hocoma, Zurich, Switzerland) for $30 \mathrm{~min}$. All participants started RAGT speed with $1 \mathrm{~km} / \mathrm{hr}$, and $40 \%$ body weight-support. Speed was increased to a range of 2.2 to $2.5 \mathrm{~km} / \mathrm{hr}$ before body weight-support was decreased. The authors used different outcomes measures to assess the participant at baseline and after RAGT intervention. The primary outcome measurements utilized in this study included the FOG-Q, FOG, and Falls Diary and Visual FOG (vFOG). The authors reported that $20.7 \%$ reduction in average frequency of freezes per day as recorded on the FOG calendars, with three participants reporting 2-3 fewer episodes of freezing per day. In addition, they observed that $13.8 \%$ improvement on the FOG-Q from baseline to end of training and severity of freezing improved $41.7 \%$ in "overall" and "initiation" FOG, which correspond to questions 4 and 5 of the FOG-Q. Moreover, median frequency vFOG scores improved $73.2 \%$ immediately following training sessions. Additionally, median frequency vFOG scores improved $62.5 \%$ from baseline to end of training. The severity of FOG was reduced in all walking contexts for all participants from baseline to end of training. The authors reported that there were no serious adverse events related to the study (Lo et al., 2010). Barbe et al. (2013) set up a pilot study to evaluate the long-term effects of RAGT on FOG reduc- tion. Three subjects participated in this study, the median age was 64.0 years and median UPDRS III score was 31. All participants received 10-12 training sessions of 30 min on RAGT (Lokomat, Hocoma, Switzerland) in an "on" phase medication. The gait speed started at $1.5 \mathrm{~km} / \mathrm{hr}$, fully assisted leg movements and a body weight support of $70 \%$. In the training session, body weight support was initially set at $100 \%$ and was then gradually reduced to $70 \%$. The FOG assessed by FOG-Q, it's completed before and after the intervention, and also after a 6-week follow-up for evaluation of long-term effects. The authors reported that RAGT improved significantly post interventions (FOG-Q, 15.3 to 10.3) but no significant effect was observed after 6 weeks of RAGT interventions (Barbe et al., 2013).

Ustinova et al. (2011) utilized a study to assess the effect of RAGT in one patient with moderate PD. In this case study, a 67-year-old female with more than an 8-year history of PD. The participant performed six sessions for 25-40 min of RAGT (Lokomat) on their "on" state medication, each week she trained 3 times a week for 2 weeks. The patient started training with walking for $25 \mathrm{~min}$ (walking distance, $0.7 \mathrm{~km}$ ), then gradually increasing by 3 - to 4 -min increment each session to $42 \mathrm{~min}$ (walking distance, $1.2 \mathrm{~km}$ ). Body weight support was gradually reduced from $44 \%$ at the beginning of therapy to $29 \%$ by the end. RAGT performed in front of a mirror where the patient could watch her performance, supplementing somatosensory feedback from the lower extremities with additional visual cues. Items 14 in unified Parkinson disease rating scale used to assess the FOG. The authors observed a significant change in walking activities, 
with a reduced occurrence of freezing episodes post RAGT (Ustinova et al., 2011).

Pilleri et al. (2015) evaluated the effectiveness of RAGT on FOG severity and gait abnormalities in PwPD. Eighteen subjects median age 64.5 years with PD and FOG resistant to dopaminergic medications participated in this study. All participants were trained on RAGT (Rehastim, Berlin) for 30 min, in 5 days a week for 3 consecutive weeks. Walking speed was set for each patient at a comfortable velocity (range, $0.5-2 \mathrm{~km} / \mathrm{hr}$ ). Severity of FOG is assessed by FOG-Q at baseline and after the RAGT intervention. The authors found that FOG-Q score was reduced from 13 \pm 3.39 at baseline to $9.2 \pm 5.44$ after treatment $(P=0.023)$. In addition, the gait parameters and functional balance were significantly improved after RAGT interventions (Pilleri et al., 2015).

\section{Effect of RAGT on spatiotemporal gait characteristics and balance}

Spatiotemporal gait parameters represented as stride length, stride time, one and double leg support time, and cadence. Gait parameters measured by different ways in three included studies. Lo et al. (2010) used a 29-foot instrumented walkway to record these gait parameters. They reported significant improvement in gait velocity and stride length $24.1 \%$ and $23.8 \%$, respectively after RAGT. In addition, step length, stride length, swing time and stride time significantly reduced after RAGT. Barbe et al. (2013) reported also a significant increase in step length and a decrease in step length variation. Moreover, the number of steps and walking time for $6 \mathrm{~m}$ were reduced significantly after RAGT. Ustinova et al. (2011) reported a significant improvement for gait velocity in their case study after RAGT. They observed that the gait cadence increased $66 \%$, turning time decreased $56 \%$, and stride length increased $27 \%$ after RAGT.

Balance also showed significant improvement in favour of RAGT. Two studies used the UPDRS items 13-15 and 29-30 to assess gait and balance (Lo et al., 2010; Ustinova et al., 2011). Both studies reported a reduction in the UPSDRS items after RAGT, which means gait and balance improved after RAGT. Moreover, Pilleri et al. (2015) used Bearg balance scale, time up and go test and Fear of Falling Efficacy Scale to evaluate clinical measures of balance, the risk of falling, and functional mobility. They reported a significant improvement in functional balance after RAGT. These improvements of self-perceived steadiness and objective balance performances indicating a positive effect of RAGT intervention.

\section{DISCUSSION}

Our aim with this review was to evaluate the currently available published data on the use of RAGT to treat FOG in PwPD. All the 4 studies and reports reviewed noted positive outcomes with using RAGT on FOG, gait parameters and balance. All studies reported that were no adverse events or side effects that occurred during and/or after the interventions. Numerous studies also emphasized improved motor impairments, gait and balance in participants when using RAGT (Alwardat et al., 2018b; Furnari et al., 2017; Picelli et al., 2012; Sale et al., 2012).

The amelioration in FOG, gait and balance could explain by different hypothesis. The several repetitions of rhythmic limb movements could act as an external proprioceptive cue, by reinforcing the neuronal circuits that contribute to the lower limb movements (Nieuwboer et al., 2009). Additionally, proprioceptive cues have been proposed to share the same mechanism of visual and auditory cues, so it is plausible that they might be involved in the gait improvements observed in PwPD after RAGT (McIntosh et al., 1997; Suteerawattananon et al., 2004). In line with this suggestion, we argue that RAGT could improve FOG and gait function in PwPD. The use of RAGT may improve compliance due to its ability to make therapy sessions more enjoyable. The increasing availability, low cost, and safety of RAGT systems further reinforces the use of RAGT in the setting of PD rehabilitation (Alwardat et al., 2018b).

However, despite the predominately beneficial effects of RAGT noted, the quality of evidence of current literature remains low. The studies were predominantly case reports and the remaining varied in size and outcome measures; therefore, it was not possible to compare and pool data for formal meta-analysis. Moreover, the included studies in this review performed the assessments and training during usual medication ('ON' medication). Assessment of motor impairments without medication ('OFF' medication) is important because the patients experienced more difficulties in motor function than on-medication whereas the symptoms are satisfactory controlled with medication. Nevertheless, despite these limitations, the reported studies show some encouraging findings that would deserve to be investigated by means of larger randomized controlled trials with standardized treatment protocols.

In conclusion, overall, while generally supportive, only very limited evidence is available to support the use of RAGT to treat FOG in PwPD. As a rehabilitation modality, RAGT has the potential and feasibility to provide significant therapy options for 


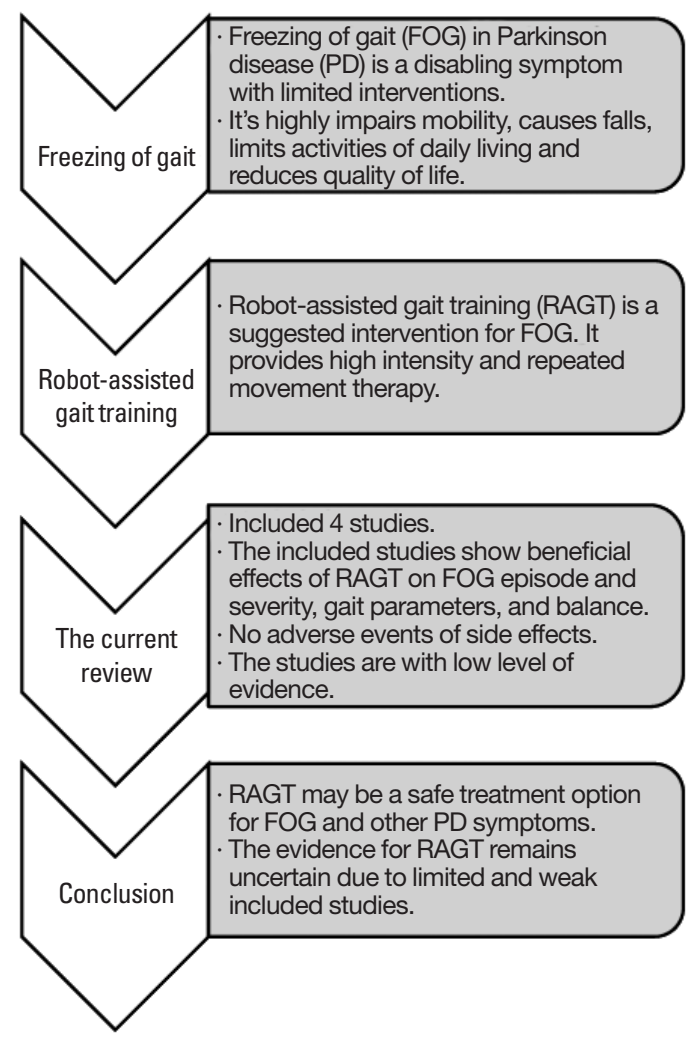

Fig. 1. The summary of the study.

PwPD and FOG, yet remains to be fully investigated at this time (Fig. 1).

\section{CONFLICT OF INTEREST}

No potential conflict of interest relevant to this article was reported.

\section{REFERENCES}

Alwardat M. Comments on: "Nordic walking for the management of people with Parkinson disease: a systematic review". PM R 2018a;10: 560-561.

Alwardat M, Etoom M, Al Dajah S, Schirinzi T, Di Lazzaro G, Sinibaldi Salimei P, Biagio Mercuri N, Pisani A. Effectiveness of robot-assisted gait training on motor impairments in people with Parkinson's disease: a systematic review and meta-analysis. Int J Rehabil Res 2018b; 41:287-296.

Barbe MT, Cepuran F, Amarell M, Schoenau E, Timmermann L. Long-term effect of robot-assisted treadmill walking reduces freezing of gait in Parkinson's disease patients: a pilot study. J Neurol 2013;260:296-298.
Bloem BR, Hausdorff JM, Visser JE, Giladi N. Falls and freezing of gait in Parkinson's disease: a review of two interconnected, episodic phenomena. Mov Disord 2004;19:871-884.

Chang WH, Kim MS, Park E, Cho JW, Youn J, Kim YK, Kim YH. Effect of dual-mode and dual-site noninvasive brain stimulation on freezing of gait in patients with Parkinson disease. Arch Phys Med Rehabil 2017; 98:1283-1290.

De Boer AG, Wijker W, Speelman JD, de Haes JC. Quality of life in patients with Parkinson's disease: development of a questionnaire. J Neurol Neurosurg Psychiatry 1996;61:70-74.

Ferraye MU, Debû B, Pollak P. Deep brain stimulation and gait disorders in Parkinson disease. Rev Neurol (Paris) 2010;166:178-187.

Frazzitta G, Maestri R, Uccellini D, Bertotti G, Abelli P. Rehabilitation treatment of gait in patients with Parkinson's disease with freezing: a comparison between two physical therapy protocols using visual and auditory cues with or without treadmill training. Mov Disord 2009;24: 1139-1143.

Furnari A, Calabrò RS, De Cola MC, Bartolo M, Castelli A, Mapelli A, Buttacchio G, Farini E, Bramanti P, Casale R. Robotic-assisted gait training in Parkinson's disease: a three-month follow-up randomized clinical trial. Int J Neurosci 2017;127:996-1004.

Giladi N, Treves TA, Simon ES, Shabtai H, Orlov Y, Kandinov B, Paleacu D, Korczyn AD. Freezing of gait in patients with advanced Parkinson's disease. J Neural Transm (Vienna) 2001;108:53-61.

Goodwin VA, Richards SH, Taylor RS, Taylor AH, Campbell JL. The effectiveness of exercise interventions for people with Parkinson's disease: a systematic review and meta-analysis. Mov Disord 2008;23:631640.

Kerr GK, Worringham CJ, Cole MH, Lacherez PF, Wood JM, Silburn PA. Predictors of future falls in Parkinson disease. Neurology 2010;75:116124.

Killane I, Fearon C, Newman L, McDonnell C, Waechter SM, Sons K, Lynch T, Reilly RB. Dual motor-cognitive virtual reality training impacts dual-task performance in freezing of gait. IEEE J Biomed Health Inform 2015;19:1855-1861.

Lo AC, Chang VC, Gianfrancesco MA, Friedman JH, Patterson TS, Benedicto DF. Reduction of freezing of gait in Parkinson's disease by repetitive robot-assisted treadmill training: a pilot study. J Neuroeng Rehabil 2010;7:51.

McIntosh GC, Brown SH, Rice RR, Thaut MH. Rhythmic auditory-motor facilitation of gait patterns in patients with Parkinson's disease. J Neurol Neurosurg Psychiatry 1997;62:22-26.

Mehrholz J, Friis R, Kugler J, Twork S, Storch A, Pohl M. Treadmill training for patients with Parkinson's disease. Cochrane Database Syst Rev 2010;(1):CD007830. 
Nieuwboer A, Rochester L, Müncks L, Swinnen SP. Motor learning in Parkinson's disease: limitations and potential for rehabilitation. Parkinsonism Relat Disord 2009;15 Suppl 3:S53-58.

Nutt JG, Bloem BR, Giladi N, Hallett M, Horak FB, Nieuwboer A. Freezing of gait: moving forward on a mysterious clinical phenomenon. Lancet Neurol 2011;10:734-744.

Pelosin E, Barella R, Bet C, Magioncalda E, Putzolu M, Di Biasio F, Cerulli C, Casaleggio M, Abbruzzese G, Avanzino L. Effect of group-based rehabilitation combining action observation with physiotherapy on freezing of gait in Parkinson's disease. Neural Plast 2018;2018:4897276.

Picelli A, Melotti C, Origano F, Waldner A, Gimigliano R, Smania N. Does robotic gait training improve balance in Parkinson's disease? A randomized controlled trial. Parkinsonism Relat Disord 2012;18:990-993.

Pilleri M, Weis L, Zabeo L, Koutsikos K, Biundo R, Facchini S, Rossi S, Masiero S, Antonini A. Overground robot assisted gait trainer for the treatment of drug-resistant freezing of gait in Parkinson disease. J Neurol Sci 2015;355:75-78.

Sale P, Franceschini M, Waldner A, Hesse S. Use of the robot assisted gait therapy in rehabilitation of patients with stroke and spinal cord injury. Eur J Phys Rehabil Med 2012;48:111-121.

Schaafsma JD, Balash Y, Gurevich T, Bartels AL, Hausdorff JM, Giladi N. Characterization of freezing of gait subtypes and the response of each to levodopa in Parkinson's disease. Eur J Neurol 2003;10:391-398.

Suteerawattananon M, Morris GS, Etnyre BR, Jankovic J, Protas EJ. Effects of visual and auditory cues on gait in individuals with Parkinson's disease. J Neurol Sci 2004;219:63-69.

Ustinova K, Chernikova L, Bilimenko A, Telenkov A, Epstein N. Effect of robotic locomotor training in an individual with Parkinson's disease: a case report. Disabil Rehabil Assist Technol 2011;6:77-85. 